\title{
Design and Development of a Mechanism for Lower Limb Movement
}

\author{
Francesco Aggogeri, Alberto Borboni, Nicola Pellegrini and Riccardo Adamini \\ Department of Mechanical and Industrial Engineering, University of Brescia, Brescia, Italy \\ Email: francesco.aggogeri@unibs.it,nicola.pellegrini@unibs.it
}

\begin{abstract}
Robotics for human rehabilitation consents to respond on open challenges and opportunities to integrate engineering concepts into human service. This paper presents a modular and reconfigurable robotic device focused on ankle rehabilitation tasks. The proposed configuration guarantees the rehabilitation progression and torque assistance during the ankle exercise, avoiding any potential disturbance. The design has been guided by patients' requirements and ankle motion. The kinematic, dynamic analyses and the mechatronic model are proposed. The hardware in the loop strategy was adopted and validated using a number of simulations. Finally, the device was validated through a set of experimental tests. The obtained results confirmed the theoretical and simulation models, thereby highlighting the potential effectiveness of the proposed system for ankle rehabilitation.
\end{abstract}

Index Terms - robotic device, kinematic analysis, applied mechanics, control strategy

\section{INTRODUCTION}

Medical treatments aim to augment the motor functionalities of limbs [1, 2]. State of the art has demonstrated that a subject may progress the own motion dysfunction with a rehabilitation program [3-5]. The therapy is so successful as it sustains the joints movement through passive training activities [6]. The errorless learning is an usual application of rehabilitation-driven treatment. In this way, the application of robots for rehabilitation and functional improvements has produced an increasing interest of scientific community. Robotassisted strategies may provide a number of advantages guaranteeing the range of motion and avoiding inappropriate movements. This study presents a prototype for ankle rehabilitation. It has been designed focusing on the integration of human joint movements with machine architecture for robot rehabilitation. Starting from the patients' requirements and an assessment of ankle motion analysis, this paper introduces the kinematics, the motor models and the control keys for the device progress. In any robotic rehabilitation equipment, the changes in joint motion are taken into account considering the structure motion and the desired increased range of ankle motion that can be fulfilled through the use of a device.

Smart therapy automaton with precise limb training features is required in stroke rehabilitation context.

Manuscript received November 9, 2018; revised July 2, 2019.
Numerous procedures and prototypes have been industrialized and can be allocated in walking and joint mechatronic systems. For example, Lokomat [7] architecture comprises a gait rehabilitation, a patient weight support and a treadmill. Alex device [8] is a motorized orthosis with hip and knee actuators, whose force regulator furnishes support to the patient as needed for gait training. Lopes exoskeleton [9] is an active exoskeleton machine that can perform specific gait exercises. In this way, a further device is Pneumatically Operated Gait Orthosis (POGO) [10] that is based on pneumatic cylinders on each leg positioned between the pelvis and ankle joint and ReWalk [10] wearable system powered by a backpack battery. It is controlled by a simple wrist-mounted remote which detects and enhances the user's movements. An alternative rehabilitation system field focuses on joints movement. NeXOS [11] is customized for home-users and his peculiarity is the webbased approach, TEM [11] is a specific device for range of motion in hip/knee joints. KAFO is a pneumatically power-driven system [12], which permits the tailored gait training with electric control. An active knee recovery machine can furnish variable force by electrorheological fluid as described in [13]. Another knee mechanism, Akrod showed by [14] research, is based on a linear motor and kinematic methodology. Other interesting prototypes are reported by Saglia et al [15]. The system for ankle rehabilitation with 2 degrees of freedom (DOF) foot pedal can achieve dorsi-plantar/flexion and inversion/eversion of ankle exercises under position control for patient-passive exercise mode or under admittance control for patient-active excise mode. Rutgers University [16] matured a 6 DOFs ankle rehabilitation robot based on Stewart platform driven by pneumatic actuation, where a force instrument was used to setting torque feed-back generated between patient foot and the foot pedal. Yu et al [17] shown a 3 DOFs cabledriven parallel automaton for ankle therapy. The mechanism design ensured that the mechanism can fit the ankle axes and rotations. Girone et al [18] elaborated a mechanism based on Stewart platform that permits an assisted ankle rehabilitation using two pneumatic actuators and torque sensors. Yano et al presented a legshaped robot of two 2 DOF manipulators equipped with footpads that guarantee movement of feet $[19,20]$. Yoon et al produced a reconfigurable ankle machine to cover several recovery isometrics tasks [21]. Instead, Dai et al 
introduced parallel devices with a dominant support for ankle [22]. Moreover a number of exoskeletal structure were matured. Jeffrey et al [23] built-up a powered ankle foot orthosis. Delaware University [24] industrialized a wearable exoskeletal ankle rehabilitation robot to assist dorsiflexion/plantarflexion and inversion/eversion training. Hong et al [25] introduced a 3 DOFs ankle rehabilitation robot, which consisted of the parallel serial chain to assist dorsiflexion/plantarflexion, and inversion/eversion training. The summary of the existing state-of-the-state rehabilitation machines can be found in following table 1. State-of-the-art highlights a broad range of valid solution. Nevertheless there are still many points to be investigated to optimize the trade-off between therapy effectiveness and system automatization. One of above mentioned open points is the control effect.

Many control methodologies are utilized for rehabilitation machine. First requirement is the design of a position or force regulator, needed to present compliance into human-robot interaction [26]. This automaton has the task of guiding limbs in a predefined trajectory [27]. Another control technique is used to synthetize the trajectory in a orthosis [28]. The subject's limb is guided on aimed trajectories. Taking the benefit from the control precision and reaction, a robot can achieve active-passive movement [29]. Authors intend to present an economical and detailed controlled mechanism with satisfying performance on limb joint therapy. Through the implementation of mechatronic knowledge, an electrical actuated mechanism is designed for precise ankle recovery.

TABLE I. State-OF-THE-STATE RehabiLitATION MAChines

\begin{tabular}{|c|c|c|c|}
\hline $\begin{array}{c}\text { Rehabilitation } \\
\text { scope }\end{array}$ & $\begin{array}{l}\text { Structure } \\
\text { outline }\end{array}$ & Actuator Type & References \\
\hline \multirow{5}{*}{ Gait Robots } & \multirow{4}{*}{ Treadmill } & \multirow{3}{*}{$\begin{array}{l}\text { Electrical } \\
\text { motor }\end{array}$} & [7] \\
\hline & & & [8] \\
\hline & & & [9] \\
\hline & & $\begin{array}{l}\text { Pneumatic } \\
\text { motor }\end{array}$ & [10] \\
\hline & $\begin{array}{l}\text { Wearable } \\
\text { devices }\end{array}$ & $\begin{array}{l}\text { Electrical } \\
\text { motor }\end{array}$ & [10] \\
\hline $\begin{array}{c}\text { Rehabilitation } \\
\text { scope }\end{array}$ & $\begin{array}{c}\text { Structure } \\
\text { outline }\end{array}$ & DOFs & References \\
\hline \multirow{15}{*}{$\begin{array}{c}\text { Joint Training } \\
\text { Robots }\end{array}$} & \multirow{12}{*}{$\begin{array}{l}\text { Parallel } \\
\text { structure }\end{array}$} & \multirow{5}{*}{ Multi } & [11] \\
\hline & & & [12] \\
\hline & & & [13] \\
\hline & & & [14] \\
\hline & & & [15] \\
\hline & & 2 & [16] \\
\hline & & 6 & [16] \\
\hline & & 3 & [17] \\
\hline & & 6 & [18] \\
\hline & & 2 & {$[19,20]$} \\
\hline & & \multirow{2}{*}{ Multi } & [21] \\
\hline & & & {$[22]$} \\
\hline & \multirow{3}{*}{ Exoskeleton } & 1 & [23] \\
\hline & & 2 & [24] \\
\hline & & 3 & [25] \\
\hline
\end{tabular}

As the ankle moves in sagittal plane, one degree of freedom (DOF) is developed but it is still adequate for the majority of the scenarios. In order to increase the awareness of system and cooperating perturbation between human-machine and to achieve the requested performance, an adaptive regulator is validated. Besides, the assembly can be tailored to the diverse statures of patients. Task of this prototype is ensuring that patients are able to fulfil joint training or desired trajectory tracking focusing on ankle joints.

\section{MOdUlaR AND RECONFIGURABLE MECHANISM}

Safety is one of the most important requirements in the automated rehabilitation. For this reason, it is essential to take into account the maximum range and maximum speed of joints flexion. The specifications are considered as boundaries of the desired trajectory, as they must ensure that motion and speed will not exceed users sustainable level. The constraints for robot control in terms of movement range of motion are for hip flexion from -1.0 to 1.0 radiant, for knee from -1.2 to -0.1 radiant and for ankle flexion from -0.9 to 0.6 radiant [9, 30, 31]. The proposed solution, able to perform the desired trajectory, is shown in Fig. 1. The modular configuration is composed by motor, electronic system, signal instrumentation and control architecture, plus mechanism which includes an auxiliary part, necessary to adjust to the diverse heights of patients. In proposed solution, the patient is seated in front of the device, due to the focus on ankle rehabilitation task. The modularity of solution allows setting the system in many different manner in order to respond to further therapies, e.g. gait rehabilitation with patient suspended, knee rehabilitation with patient supine.

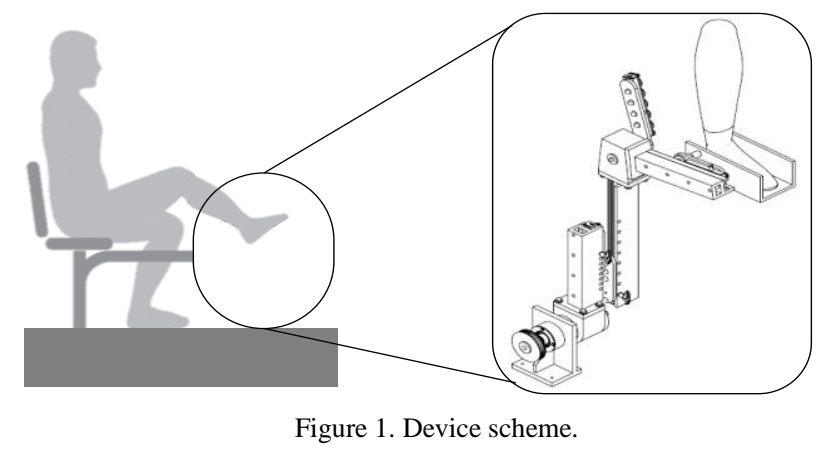

The actuator is a direct current (DC) motor and gearbox. The gearbox is connected to DC motor to extend torque and decrease the speed rotation. Precise voltages provided by motor drivers can control DC actuator by adapting the module of PWM (Pulse-Width-Modulation). The signal processing instrument consist of current sensor components, optical encoders and a data acquisition element. The first ones permit the measurement of the current of DC motor, composed by a standard $0.01 \mathrm{ohm}$ resistor and a precise instrument amplifier. The second ones measure the real-time position of the DC motor and in this way the position signal estimates the speed of motors. The usage of a commercial data acquisition system is needed, so that data can be acquired by the closed-loop. The data acquisition card controls the value of speed and current and sent them to controller for real time feedback. In this way PC is able to run the control 
strategy and to grant a human-robot interaction for therapist and subject. The functional connections of system components are illustrated in Fig. 2.

The starting point is represented by the therapist tasks to measure and set the rehabilitation trajectory using the interaction interface on PC. Then medical supervisor defines the range of motion exercise or gait physical activity and the rehabilitation database parameters like the joint angle and time of task [32]. At that point, the robot system is working and each chosen joint follows the desired trajectory.

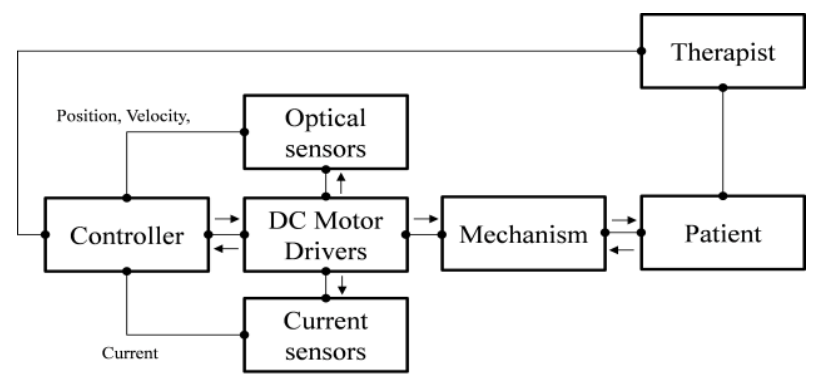

Figure 2 System description.

The output of actuator is accurately adjusted, when motor drivers control DC motors. Inputs of actuator drivers are pulse width modulations determined by control approach and sent to actuator drivers by the data acquisition element. The velocities and positions of actuators are measured by the photosensitive encoders as long as the currents are estimated by the current sensor modules. The data of velocity, position and current are monitored and these feed-back are sent to control scheme for the adjustment of pulse width modulation. Thus, a closed loop control structure is setup entirely.

\section{MODELING AND VERIFICATION}

According to the proposed solution a mathematical model was developed. The complete architecture was composed by kinematic, motor and control perspective. Kinematic scheme explains the linkage among the mechanism and ideal joint trajectory. Aim of the motor model is to describe the principle of energy conversion from electricity to kinetic energy. In conclusion, the control model drives the correction to servo actuated device from the effect of perturbations.

\section{A. Kinematic and Dinamyc Model}

There are several techniques for dynamic formulation of multi rigid machine: Lagrange, Newton-Euler, Kane and Hamilton concept. The most used ones for controller design are Lagrange and Newton Euler [33-36]. Lagrange formulation is a derivation of Newton-Euler theory but concentrates on the study of energy of the full structure while the aim of Newton-Euler equation is to analyze the component. The proposed mechanism has been synthetized on three segments representing the foot system (from hallux to ankle joint, $\phi_{3}$ ), the leg (from ankle to knee $\phi_{2}$ ) and thigh (from the knee to the hip $\phi_{1}$ ). Fig. 3 highlights the way in which the anatomical angles of joints in the sagittal plane can vary during the gait cycle. In particular they are a function of the gait cycle. Angle and payload constraints are used to determine the specified workspace boundary

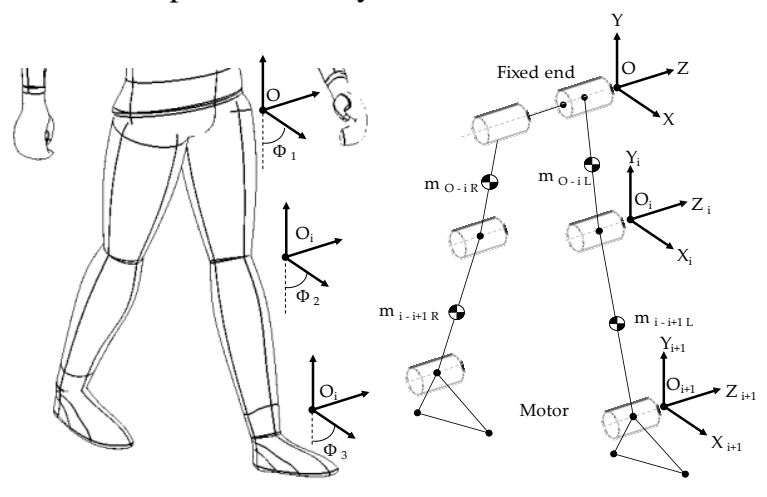

Figure 3. Linkage model

Authors selected the method of Lagrange to found the kinematic prototypical of proposed mechanism. Since the principal movement occurs in the sagittal plane, only kinematic representation in this plane is subjected to analysis. Lagrange equation is given by:

$$
\begin{aligned}
& \mathrm{LF}=W_{e}-W_{p} \\
& T_{s}=\frac{d}{d t}\left(\frac{\partial L}{\partial \dot{\phi}_{i}}\right)-\left(\frac{\partial L}{\partial \phi_{i}}\right)
\end{aligned}
$$

Where We stands for the kinetic energy of structure; $\mathrm{W}_{\mathrm{p}}$ represents all the potential energy and the Lagrange Function LF. $\phi_{\mathrm{i}}$ is the general position and $\phi_{\mathrm{i}}^{\prime}$ the speed. $\mathrm{T}_{\mathrm{s}}$ is the junction torque. $\mathrm{W}_{\mathrm{e}}$ and $\mathrm{W}_{\mathrm{p}}$ can be expressed as:

$$
\begin{aligned}
& W_{e}=\sum_{i=1}^{3} m_{i} \cdot g \cdot y_{i} ; \\
& W_{p}=\sum_{i=1}^{3}\left[\frac{1}{2} \cdot m_{i} \cdot\left(x^{2}{ }_{i}+y^{2}{ }_{i}\right)+\frac{1}{2} \cdot I_{i} \cdot \phi^{2}{ }_{i}\right] ;
\end{aligned}
$$

where $m_{i}$ represents the mass of connection; $I_{i}$ stands for the inertia of rotation; $g$ is the gravity, and $\left(x_{i}, y_{i}\right)$ is the center of gravity position of connection $i$-th. The stationary basis, $\mathrm{O}(0,0)$ represented in Fig. 3 is adopted as coordinate origin. In line with the geometric function, the coordinate of position $\left(\mathrm{x}_{\mathrm{i}}, \mathrm{y}_{\mathrm{i}}\right)$ of each connection can be stated as

$$
\left\{\begin{array}{l}
x_{i}=\sum_{j=1}^{i-1}\left(l_{j} \cdot \sin \phi_{j}\right)+d_{i} \cdot \sin \phi_{i} \\
y_{i}=\sum_{j=1}^{i-1}\left(l_{j} \cdot \cos \phi_{j}\right)+d_{i} \cdot \cos \phi_{i}
\end{array} ;\right.
$$

where $d_{i}$ represents the interval between gravity position to $\mathrm{L}_{\mathrm{i}}$ of linkage and $\phi_{\mathrm{i}}$ the angle from linkage and vertical axis. Furthermore, the derivate of position can be written as

$$
\left\{\begin{array}{c}
\dot{x}_{i}=\sum_{j=1}^{i-1}\left(l_{j} \cdot \dot{\phi}_{j} \cdot \sin \phi_{j}\right)+d_{i} \cdot \dot{\phi}_{i} \cdot \sin \phi_{i} \\
\dot{y}_{i}=\sum_{j=1}^{i-1}\left(l_{j} \cdot \dot{\phi}_{j} \cdot \cos \phi_{j}\right)+d_{i} \cdot \dot{\phi}_{i} \cdot \cos \phi_{i}
\end{array} ;\right.
$$


Combining the equation (1) to (6), the kinematic analysis in vertical plane is shown:

$$
M(\phi) \cdot \ddot{\phi}+C(\phi, \dot{\phi}) \cdot \dot{\phi}+G(\phi)=T_{s} ;
$$

where $\phi_{\mathrm{i}}^{*}$ is the angular acceleration; $\mathrm{M}(\mathrm{t})$ is the symmetrical mass vector, $\mathrm{C}$ is the centripetal force vector, $\mathrm{G}(\mathrm{t})$ stands for the gravitational force vector.

In this paper the kinematics is related to ankle on the plantar-flexion movement with the aims of increasing the degree of patients' mobility. A trajectory learning compares the kinematics of the ankle obtained with the articulated mechanism to the healthy subject. It is necessary to associate the $\mathrm{x}-\mathrm{y}$ axis to the step cycle of the patient, in time scale, as shown in Fig. 4. To describe this particular trajectory was essential to model the foot system as a triangle with vertices at the hallux, ankle and heel position. At that time it was required to define two angles: $\lambda$ - the angle from the ankle to hallux; and $\varphi-$ the angle from ankle to heel. The expectations for walk trajectory planning is based on $\lambda$ and $\varphi$ angles as independent to patients and step distance [37]. It is possible to measure the distance between ankle-hallux and ankle-heel of a patient in order to estimate the step trajectory. An iterative process is developed in order to find the most advantageous mechanism design configuration [38-43].

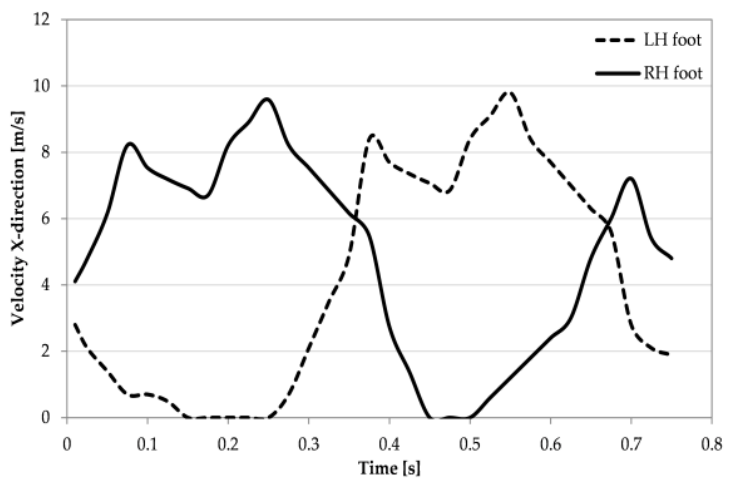

(a)

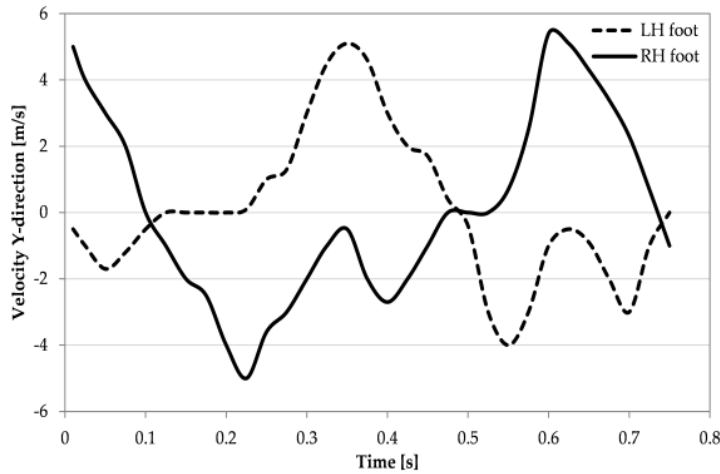

(b)

Figure 4. Reference trajectory of hallux motion in X-direction (a) and Y-direction (b)

The structure in Fig. 5 is the solution of the iterative optimization phase. In addition the proposed architecture allows the usage of one motor with the aim of performing the movement of the articulated system.

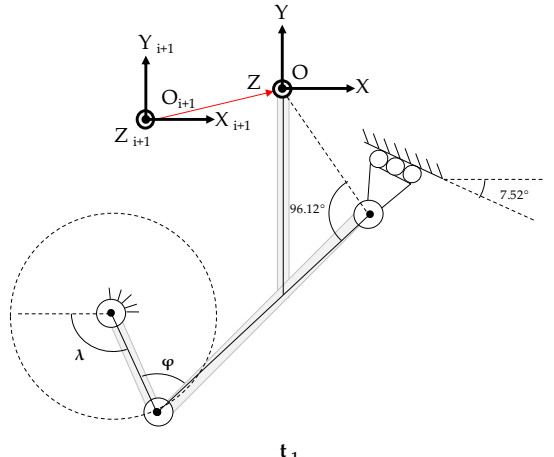

(a)

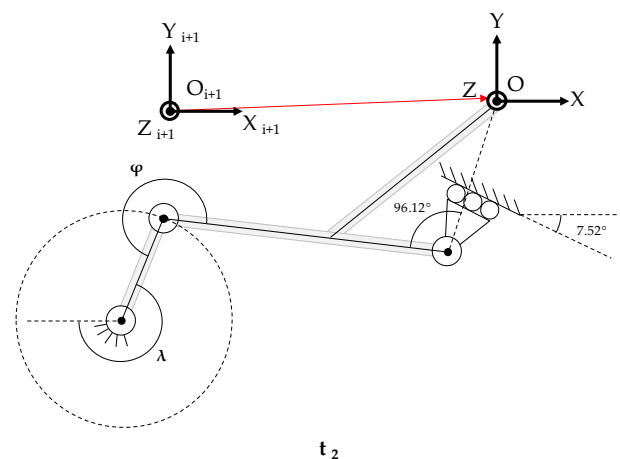

(b)

Figure 5. the mechanism in instant $\mathrm{t} 1$ (a) and $\mathrm{t} 2$ (b)

A set of conventions has been recognized in quantifying the device parameters. For example, the rehabilitation walking step cannot exceed $555 \mathrm{~mm}$ in order to guarantee patient's safety and therapy effectiveness, as extrapolated from gait analysis.

\section{B. Motor Model}

The mathematical model development was improved with the actuator side. The output torque $\mathrm{T}_{\mathrm{m}}$ of motor is directly correlated to the feeding current

$T_{m}=S_{m} \cdot i_{m}$

where $S_{m}$ represents the torque reaction and im the feeding current. According to (8), the relationship between $V_{m}$ and $T_{m}$ can be formulated as:

$\dot{T}_{, m}=-A \cdot T_{m}+S_{m} \cdot V_{m}-S_{m} \cdot F_{v} \cdot \omega$

where $A$ is the resistance, $F_{v}$ is the electromotive force, $\omega$ is the angular velocity.

The generated model permits the measurement of the motor current, to obtain the output torque. Through the measured data of angular velocity, the control can operate properly. On the base of equation (9) it is possible to build a model in Simulink SW, as represented in Fig. 6.

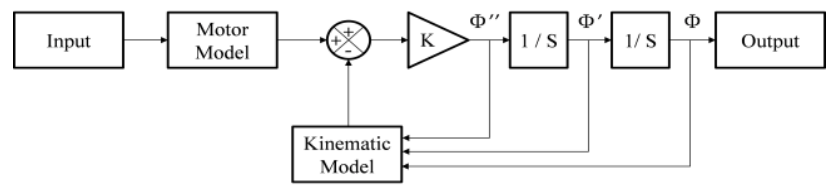

Figure 6. Control strategy scheme 
In the preliminary trials, motors receive as inputs a group of step control voltage $\mathrm{V}_{\mathrm{m}}$ and the actual angles of joint are recorded. In order to perform Hardware in the Loop validation the same control voltages are sent to Simulink model and results are recorded. The outcomes are tracking error less than $15 \%$, the steady state discrepancies from $5 \%$ to $10 \%$ mainly caused by the friction characteristics, which need to be managed by control architecture. In addition, for the duration of rehabilitation training the mechanism requires to overcome the reaction force of patient as the movement of rehabilitation system is influenced by this unknown disturbance. For this reason, a modular and adaptive control strategy is useful to reduce the impact of variability and suppress noise from patient.

\section{Sensitivity Analysis of Mechanism}

The understanding of the mechanism configuration is of great importance for the precise trajectory for human ankle rehabilitation. The main parameters investigated are: crank length, connecting rod length and the distance between the slider and the hallux position. In this paper, the structural parameters of ankle rehabilitation mechanism are optimized based on two function that needed to be achieved, the customized walking distance and the step hight.

The kinematic model is solved using an iterative method imposing different input parameters and analyzing the best solution in terms of trajectory deviation of the planar mechanism considering the above mentioned requirements. The software used in design phase is MECAD, able to integrate pre-processing, numerical analysis and post processing in an easy to use environment. Each parameter under investigation is here summarized with the focus of emphasize his impact on the trajectory XY-plane, considering three level of analysis (low, nominal and high). Fig. 7 shows the effect of the distance between the slider and the hallux position referred to the extremity of hallux motion during a complete walking cycle in sagittal plane.

As can be seen the mechanism that resulted from the optimization process shows significantly correspondence with the requested path boundary. The nominal value maximize the walking distance (limited at 555mm) and the step hight with the setting of $155.90 \mathrm{~mm}$.

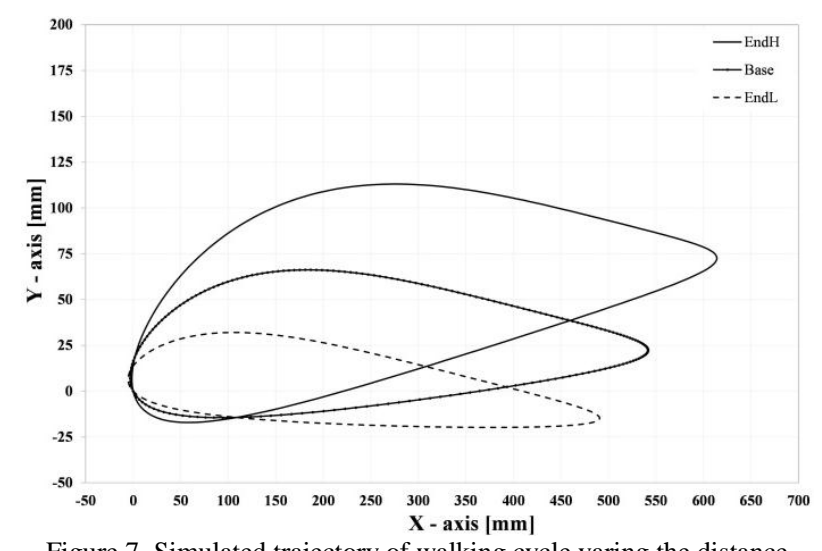

Figure 7. Simulated trajectory of walking cycle varing the distance slider-hallux
The trajectory resulting from setting $250.00 \mathrm{~mm}$ is shown with "EndH" legend, is not suitable for safety of person in rehabilitation training. The setting of $85.00 \mathrm{~mm}$ in dotted line "EndL" is not an effective solution due to the lower working space respect to the nominal setup.

Then in the Fig. 8 the sensitivity analysis was performed by the perturbation of the connecting rod, with values of $360.00 \mathrm{~mm}$ (low), $390.30 \mathrm{~mm}$ (nominal) and $500.00 \mathrm{~mm}$ (hight). In Fig. 9 the results of changing the crank length, by imposing the following inputs $100.00 \mathrm{~mm}$ (low), $191.40 \mathrm{~mm}$ (nominal) and $220.00 \mathrm{~mm}$ (hight). In the described scenarios the boundaries are fundamental in order to obtain the simulation convergence and therapy safety aspects.

In fact the connecting rod leg lower boundary is close to $350.00 \mathrm{~mm}$. Note that the crank dimension over 200.00 $\mathrm{mm}$ leads to the risk for the end user. As a final consideration, for each design, initial assembly is carried out with slider and angle in the same position to facilitate the comparison of performance.

As a final consideration, for each design, initial assembly is carried out with slider and angle in the same position to facilitate the comparison of performance.

Plots of walking length comprises average and whole variability range for the one-level at time of parameters, are presented in Figs. 10 (a), 10 (c) and 10 (e), respectively.

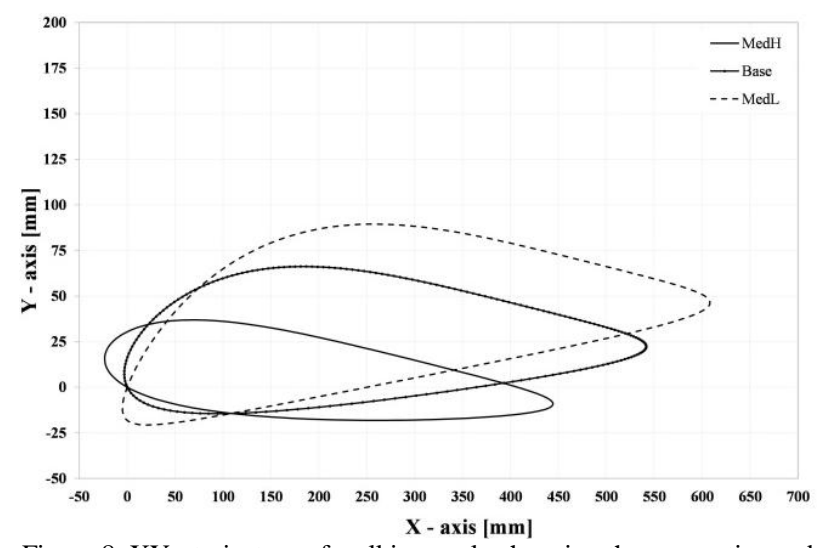

Figure 8. XY - trajectory of walking cycle changing the connecting rod length

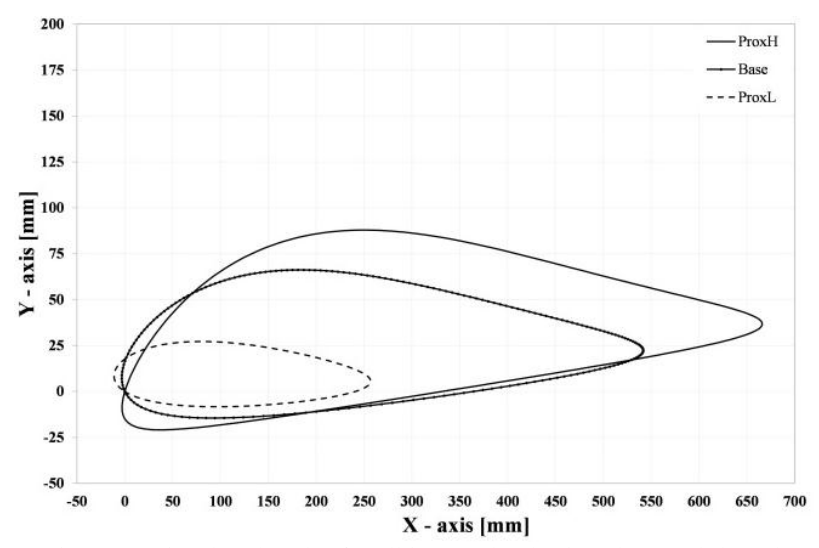

Figure 9. Simulated path of walking cycle altering the crank length 


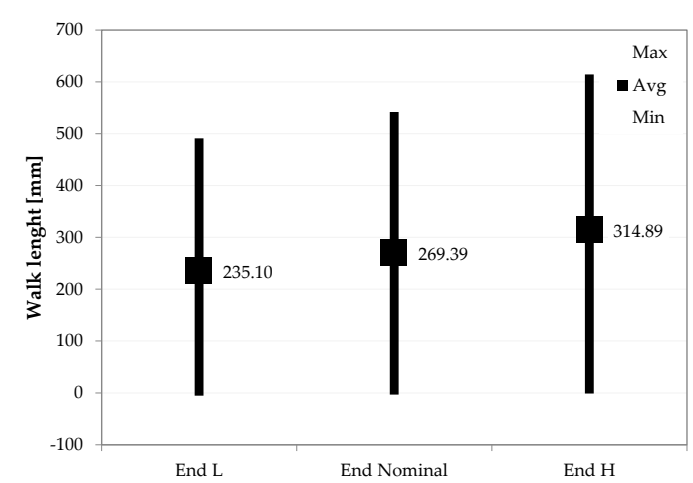

(a)

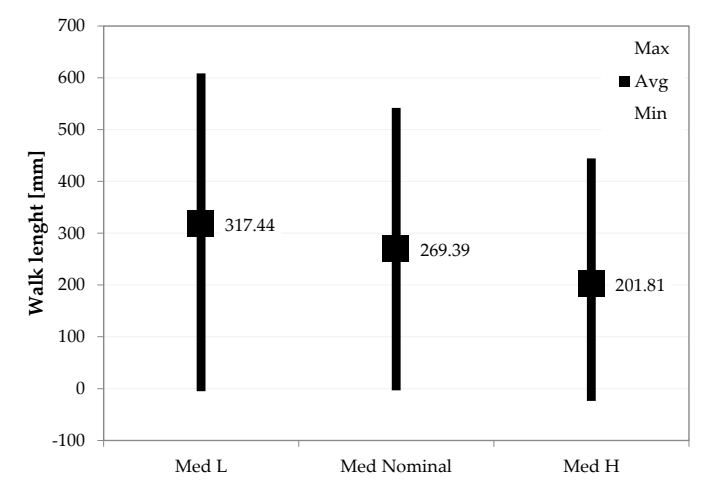

(c)

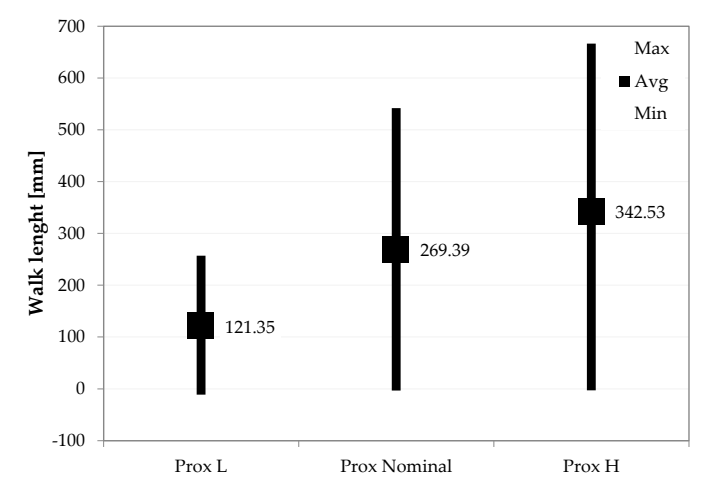

(e)

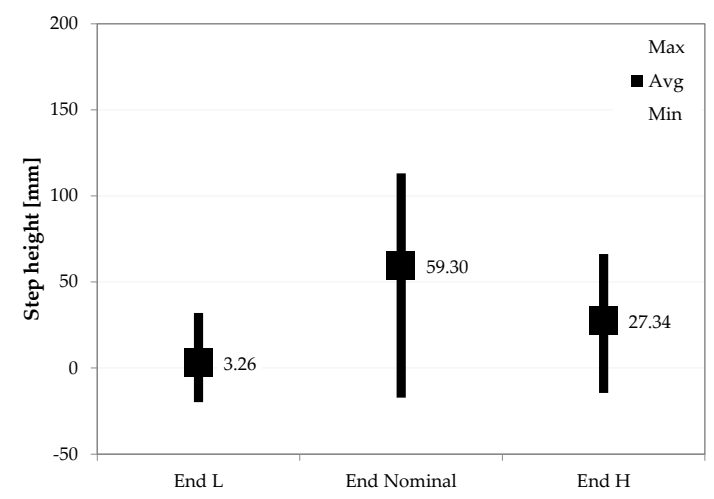

(b)

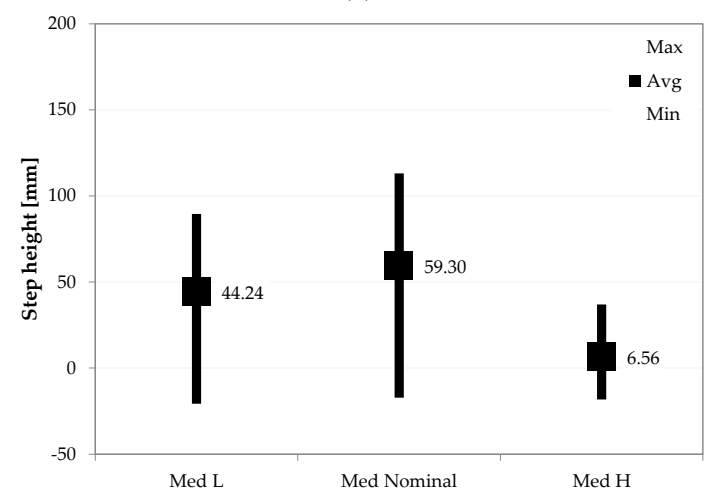

(d)

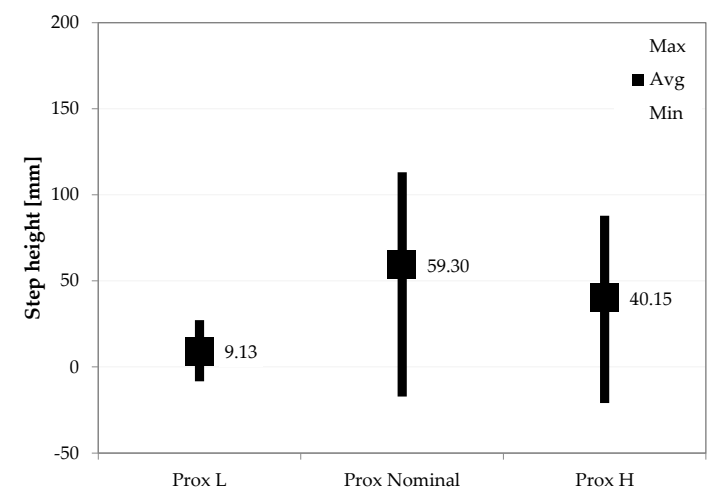

(f)

Figure 10. Walking results (a) and Vertical movement (b) of hallux motion for sliding distance variation; Walking results (c) and Vertical movement (d) of connecting rod length variation and Walking results (e) and Vertical movement (f) of crank variation
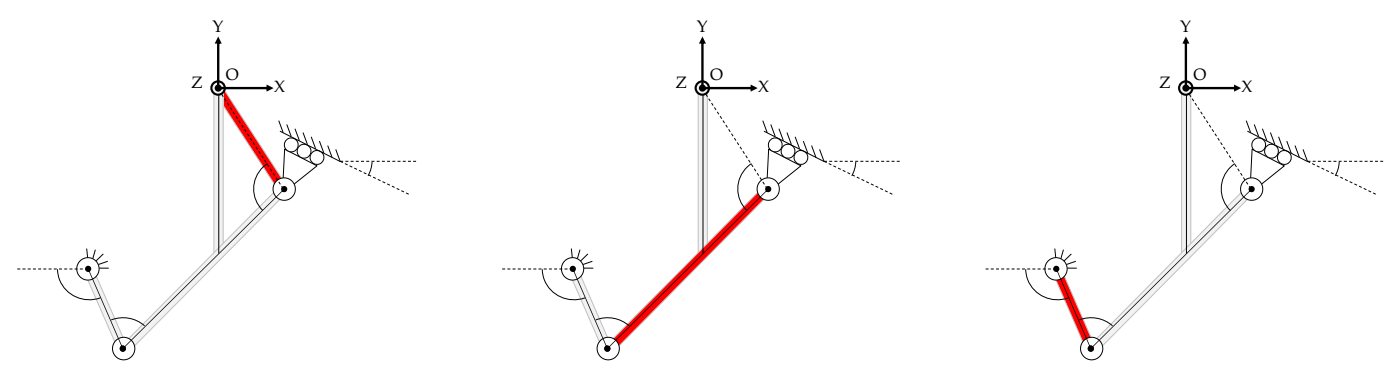

Figure 11. Parameter: Sliding distance (a), Connecting rod length (b) and Crank (c)

In order to investigate the opportunity to use the proposed mechanism for step rehabilitation the additional function that needs to be achieve is the maximization of vertical movement, with the purpose of obstacle passing or stair climbing. The vertical movement achieved by different configuration of the mechanism is reported in
Fig. 10(b), 10 (d) and 10 (f), for hallux-siding distance, connecting rod and crank, respectively, as summarized in Fig. 11 (a-c). Walking and step functions are maximized by nominal configuration of mechanism parameters as: crank length $191.40 \mathrm{~mm}$, connecting rod $390.30 \mathrm{~mm}$ and hallux-sliding distance $155.90 \mathrm{~mm}$. 


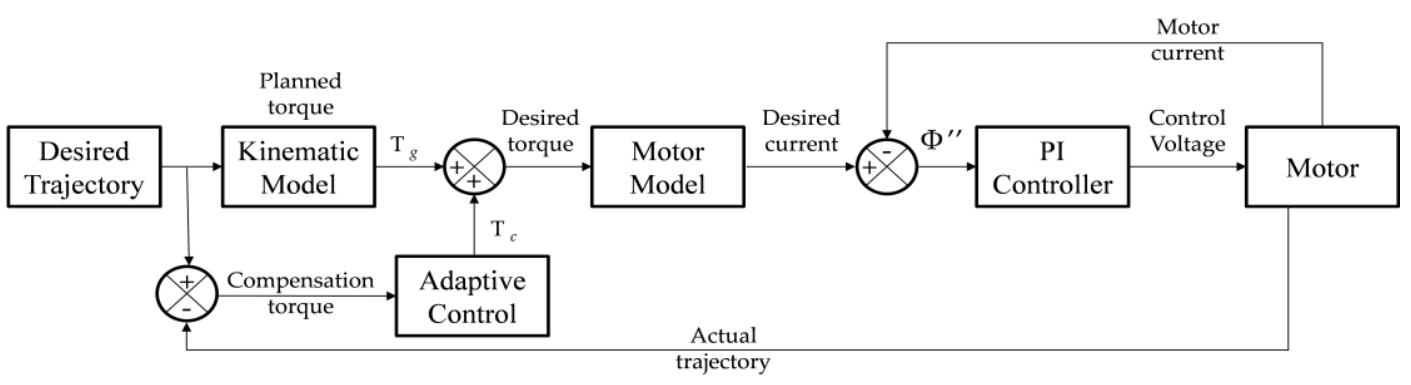

Figure 12. Control Strategy scheme

\section{ROBUST CONTROL SCHEME AND RESUlTS}

The rehabilitation mechanism needs to be controlled with satisfying performance on predetermined task activity. Therefore a regulator was synthetized to reduce mechanism tracking error and limit the noise generated by the patients.

As presented in Fig. 12 the designed torque comprises of two portions. The first one involves the planning torque of the ideal joint $T_{g}$, while in the second one, torque $T_{c}$ is considered to adjust the effect of mechanism variabilities and noise. In working conditions, regulator process includes: an reliable subcontroller to align torque $\mathrm{Tc}$ and a proportional-integrative subcontroller to create the predefined torque $\mathrm{T}_{\mathrm{md}}$. The splitting parts are the feedforward planning torque covering ideal joint torque $\mathrm{T}_{\mathrm{sd}}$ as well as the compensation torque. The definition of the trajectory error is as follows:

$$
\begin{aligned}
& E=\phi_{d}-\phi ; \\
& T_{s d}=M(\phi) \cdot\left(\ddot{\phi}_{d}+K_{d} \cdot \dot{E}+K_{p} \cdot E\right)+C(\phi, \dot{\phi}) \cdot \dot{\phi}+G(\phi) ;
\end{aligned}
$$

Taking into consideration mechanism variance, such as simulation error and external forces, uncertain item $d$ is introduced to quantify the mechanism variance.

$$
\ddot{E}+K_{d} \cdot \dot{E}+K_{p} \cdot E=-M^{-1} \cdot\left(T_{c}-d\right) ;
$$

From equation (12), authors can know that if $T_{c}-d$ is zero, and $K_{d}, K_{p}$ are appropriately selected, then error tends to zero in ideal state. With the aim of eliminating the effect of mechanism variance and disturbance $d$, adjusted torque Tc should be employed to ensure $T_{c}-d$ close to zero as for the predetermined time. Instead of smoothing the overshooting of adaptive regulator (AR) sub-controller and in order to get a more accurate control performance a proportional integrative sub-regulator is designed as

$$
V_{m}=k_{1} \cdot i_{e}+k_{2} \cdot \int i_{e} \cdot d t+K_{v} \cdot \omega ;
$$

where $\mathrm{k}_{1}, \mathrm{k}_{2}$ are proportional and integral gain, respectively; $\mathrm{V}_{\mathrm{m}}$ is the adjusting voltage. Then, based on adjusted voltage $\mathrm{V}_{\mathrm{m}}$, the resultant signals are directed to actuator drivers. To validate the regulator efficacy, a simulation campaign has been developed.

Parameters as, $\mathrm{k}_{1}, \mathrm{k}_{2}, \mathrm{~K}_{\mathrm{d}}, \mathrm{K}_{\mathrm{p}}, \lambda, \rho, \delta$ are adjusted and reviewed following the trajectory tracking performance, as summarized in Table II.
TABLE II. CONTROLler PARAMETER VALUES

\begin{tabular}{|c|c|}
\hline Parameter & Value \\
\hline $\mathbf{k}_{\mathbf{1}}$ & -0.45 \\
\hline $\mathbf{k}_{\mathbf{2}}$ & 0.01 \\
\hline $\mathbf{K}_{\mathbf{d}}$ & 15 \\
\hline $\mathbf{K}_{\mathbf{p}}$ & 56 \\
\hline $\boldsymbol{\lambda}$ & 1.5 \\
\hline $\boldsymbol{\rho}$ & 10.5 \\
\hline
\end{tabular}

The results of the verification of robust controller are fully consistent with the simulation response. The desired trajectory is aligned with the actual trajectory with minor error. The results confirm that controller can overcome the dynamics perturbation effectively. The lower limb rehabilitation system designed in this paper helps stroke patients to perform rehabilitation training, including range of motion exercise. To validate the mechanism, authors designed two experimental campaigns. In range of motion therapy, one joint is trained, the other joints are fixed. In this trial configuration, rehabilitation device works without any patient. The campaign is a fundamental confirmation of controller performance in theoretical environment. Fig. 13 (a) presents the range of motion tracking performance of Ankle joint, while Fig. 13 (b) explains the control signals and the outputs of actuator. From Fig. 13 (a) it is highlighted that actual trajectory of therapy was aligned to the desired trajectory. As shown in Fig. 13 (b), the control and output signals of joints are all periodic and have a satisfying reliability, due to the fact that the desired trajectories are periodic as well and there was no perturbation from subject for the duration of the experiment. This test confirms that the concept of rehabilitation system developed in this research is highly precise and the regulator can refine tracking activities.

For validating the purpose of rehabilitation system, a set of trials has been completed on healthy subjects. The device drives the subject to desired path and the outcome of evaluation are used to measure the performance. Experimental results of gait training are shown in Fig. 14.

Fig. 14 (a) highlights that the device has an acceptable performance in trajectory following with subject intervention. It means that subject can obtain the arranged therapy training by the assistance of this mechanism. Considering the effect of response from subject, the considered regulator wants to suppress the force and 
deviations in reaching desired trajectory in this way an increasing in performance can be achieved.

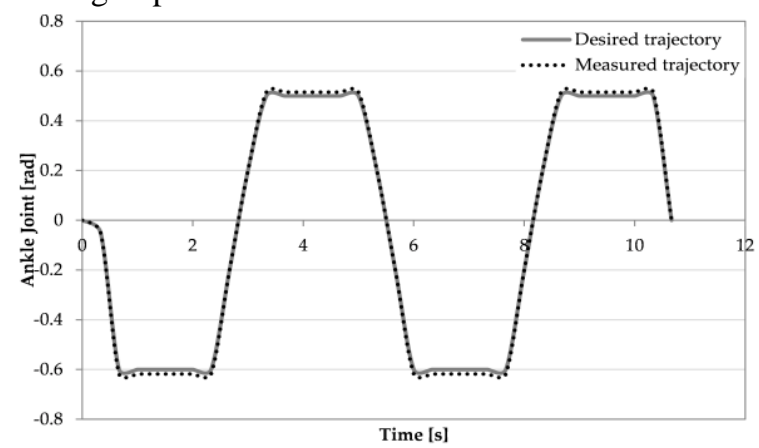

(a)

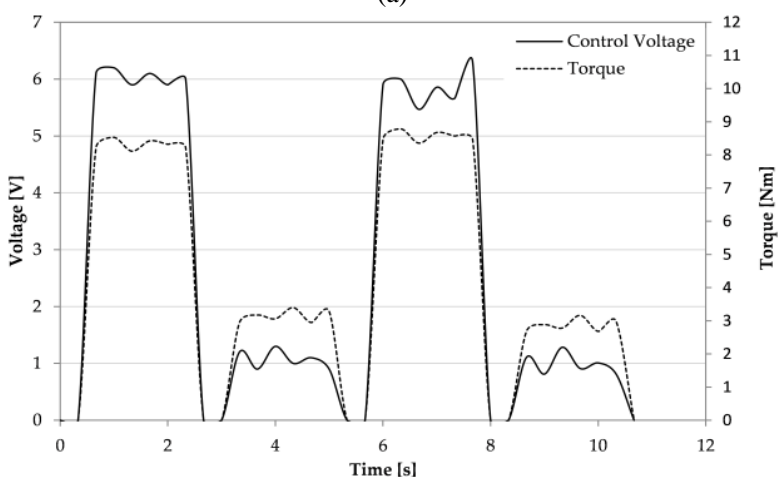

(b)

Figure 13 Results of motion - Mechanism (a) tracking performance of ankle joint; (b) control voltage and torque

Fig. 14 (b) shows that even if control signal is periodic, it is diverse in each sequence as the controller procedure is operating with the purpose of compensating force and perturbation from patient during therapy exercise. As response of subject is not constant, the control voltage should change in accordance with compensation.

In conclusion with this experiment it is possible to show that lower limb rehabilitation system considered in this research can overcome the irregularities of subject successfully.

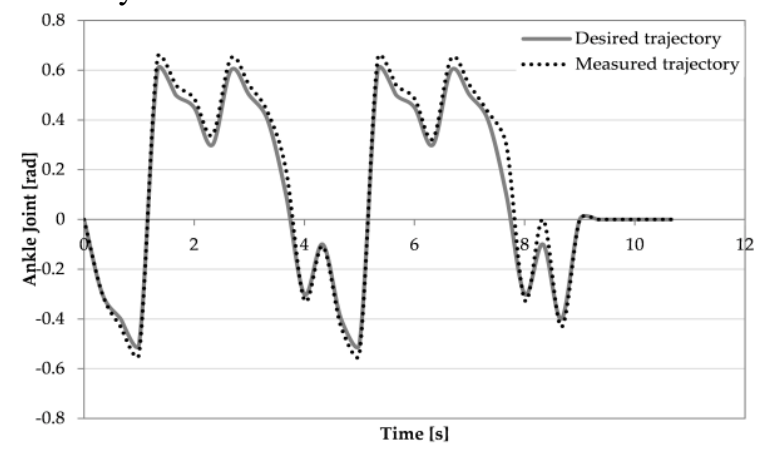

(a)

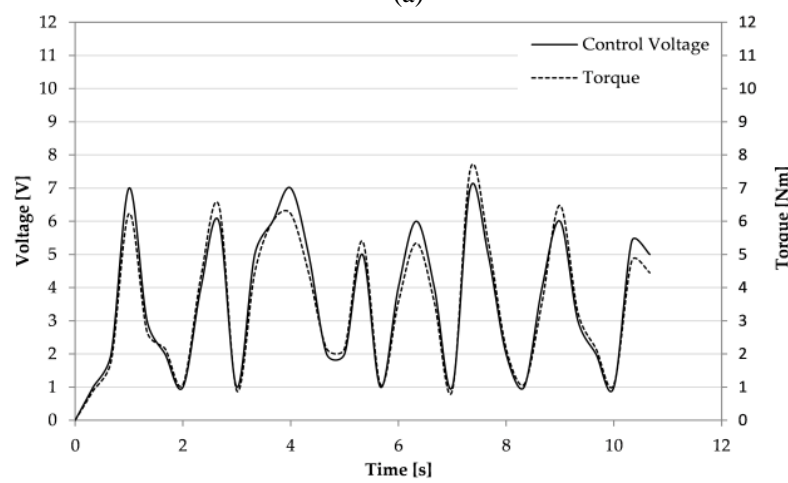

(b)

Figure 14 Results of motion- (a) tracking performance of health ankle joint; (b) control voltage and torque

Table III shows a summary of the principal benefits and restrictions of the ankle rehabilitation mechanism.

The future steps of the research will be found on improvement of device portability, refining user-friendly features, extend the rehabilitation at multi-joints at the same time. The final purpose will be to test and validate the rehabilitation device involving patients, physiotherapists and physicians.

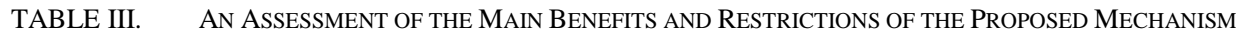

\begin{tabular}{|c|c|}
\hline Benefits & Margins \\
\hline $\begin{array}{l}\text { Robot is suitable for different patients: } \\
\text { individual customization is the adding-value due to trajectory-kinematics } \\
\text { parameters setting and actuation system selection. }\end{array}$ & $\begin{array}{l}\text { Passive Motion: } \\
\text { the system currently does not consider the three main joints of lower limb. }\end{array}$ \\
\hline $\begin{array}{l}\text { Repeatability of movement: } \\
\text { the system architecture based on a parallel mechanic can guarantee } \\
\text { precision of movement while the patient's body remains stationary }\end{array}$ & $\begin{array}{l}\text { A friendly interface for robot user: } \\
\text { A touch panel, will enable robot user easily enter the parameters and } \\
\text { instructions. A portable device allows user to set parameters at a } \\
\text { comfortable location. }\end{array}$ \\
\hline $\begin{array}{l}\text { Adaptive control: } \\
\text { the rehabilitation mode brings joint training and gait training with easily and } \\
\text { accurate control of each joint and avoids the overload. Usually the } \\
\text { rehabilitation robots are position-based control, while this innovative } \\
\text { control method is structure-based to provide torque assistance. }\end{array}$ & $\begin{array}{l}\text { Integration with virtual reality scenarios: } \\
\text { Patient will potentially perform ankle training under awareness exercise } \\
\text { mode, a virtual training scenario integrating with patient rehabilitation } \\
\text { information will stimulate the robot user to concentrate on training and } \\
\text { make the rehabilitation become an attractive activity. }\end{array}$ \\
\hline
\end{tabular}




\section{CONCLUSION}

This paper presents a reconfigurable single DOF system with a designed and a validated controller. The mechanism focuses on ankle joint, and can be adapted to the connections for fitting and customizing the subject dimensions. Using a modular and flexible configuration a rehabilitation system is able to provide and guarantee the rehabilitation cycle and passive torque assistance during the required therapy.

The mechatronic model is composed by kinematic and motor perspective. Authors studied and validated the hardware in the loop using SW modelling and trials, demonstrating that the concept highly considers the mechanism dynamics. The suggested controller is based on planning torque in feedforward network as long as an adaptive procedure is synthetized in the feed-back loop to reduce the mechanism deviations and perturbation from end-user subject. Using software simulations the ankle motion is successfully demonstrated by the rehabilitation system.

In the described campaigns, rehabilitation exercises are executed with the aim of testing controller performance with and without subject reaction.

In conclusion the system performs rehabilitation tasks with acceptable tracking error in passive education of movement. The possibility to include analysis performed on patients should improve the dynamic control and the clinical effectiveness quantifying the ability and the progress during training process.

\section{REFERENCES}

[1] A. Mirelman, P. Bonato, J. E. Deutsch, "Effects of training with a robot-virtual reality system compared with a robot alone on the gait of individuals after stroke," Stroke, vol. 40, no. 1, pp. 169-174, 2009.

[2] A. Lamontagne, J. Fung, "Faster is better - implications for speed intensive gait training after stroke," Stroke, vol. 35, no. 11, pp. 2543-8, 2004

[3] G. T. Thielman, C. M. Dean, A. M. Gentile, "Rehabilitation of reaching after stroke: Task-related training versus progressive resistive exercise," Arch Phys Med Rehabil, vol. 85, no. 10, pp. 1613-18, 2004.

[4] K. Probosz, R. Wcislo, J. Otfinowski, R. Slota, J. Kitowski, M. Pisula, etal. "A multimedia holistic rehabilitation method for patients after stroke," Cyber psychol Behav, vol. 12, no. 5, pp. 646-7, 2009.

[5] S. P. Johnsen, L. P. Kammersgaard, "Increasing incidence of hospitalization for stroke and transient ischemic attack in young adults: A registry-based study," Journal of the American Heart Association, vol. 5, no. 5, May 2016.

[6] D. Bradley, C. Acosta-Marquez, M. Hawley, S. Brownsell, P. Enderby, S. Mawson. "NeXOS - The design, development and evaluation of a rehabilitation system for the lower limbs," Mechatronics, vol. 19, pp. 247-257, 2009.

[7] R. Riener, L. Lunenburger, S. Jezernik, M. Anderschitz, C. Colombo, V. Dietz, "Patient-cooperative strategies for robot-aided treadmill training: first experimental results," IEEE Trans Neural Syst Rehabil Eng, vol. 13, no. 3, pp. 380-94, 2005.

[8] S. K. Banala, S. H. Kim, S. K. Agrawal, J. P. Scholz, "Robot assisted gait training with active leg exoskeleton (alex)," IEEE Trans Neural Syst Rehabil Eng, vol. 17, no. 1, pp. 2-8, 2009.

[9] J. F. Veneman, R. Kruidhof, E. E. G. Hekman, R. Ekkelenkamp, E. H. F. Van Asseldonk, H. Vander Kooij, "Design and evaluation of the lopes exoskeleton robot for interactive gait rehabilitation,"
IEEE Trans Neural Syst Rehabil Eng, vol. 15, no. 3, pp. 379-86, 2007.

[10] D. Aoyagi, W. E. Ichinose, S. J. Harkema, D. J. Reinkensmeyer, and J. E. Bobrow, "A robot and control algorithm that can synchronously assist in naturalistic motion during body-weightsupported gait training following neurologic injury," IEEE Transactions on Neural Systems and Rehabilitation Engineering, vol. 15, no. 3, Sep. 2007.

[11] D. Bradley, C. Acosta-Marquez, M. Hawley, S. Brownsell, P. Enderby, S. Mawson, "Nexos-the design, development and evaluation of a rehabilitation system for the lower limbs," Mechatronics, vol. 19, no. 2, pp. 247-57, 2009.

[12] G. S. Sawicki, D. P. Ferris, "Mechanics and control of a knee ankle foot orthosis (KAFO) powered with artificial pneumatic muscles," Journal of Biomechanics, vol. 39, no. 1, 2006, pp. S109.

[13] M. Nazemizadeh, M. Taheri, and S. H. Nazeri, "Dynamic modeling and path planning of flexible-link manipulators," International Journal of Mechanical Engineering and Robotics Research, vol. 3, no. 2, pp. 223-228, April 2014.

[14] K. Kim, M. Kang, Y. Choi, H. Jang, J. Han, C. Han, "Development of the exoskeleton knee rehabilitation robot using the linear actuator," Int J Precis Eng Manuf, vol. 13, no. 10, pp. 1889-95, 2012.

[15] J. Saglia, N. Tsagarakis, and J. Dai, and D. Caldwell, "A high performance 2dof over actuated parallel mechanism for ankle rehabilitation," IEEE International Conference on Robotics and Automation, Kobe, Japan, pp. 2180-2186, May 2009.

[16] A. B. Farjadian, M. Nabian, A. Hartman, J. Corsino, C. Mavroidis, M. K. Holden, "Position versus force control: using the 2-DOF robotic ankle trainer to assess ankle's motor control," Conf Proc IEEE Eng Med Biol Soc. vol. 2014, 2014:1186-9.

[17] G. Chen, P. Qi, Z. Guo, H. Y. Yu, "Mechanical design and evaluation of a compact portable knee-ankle-foot robot for gait rehabilitation," Mechanism and Machine Theory, vol. 103, September 2016, pp. 51-64.

[18] K. Manjuladevi et al. "The Rutgers arm, a rehabilitation system in virtual reality: A pilot study," Cyberpsychology \& behavior : the impact of the Internet, multimedia and virtual reality on behavior and society, vol. 9, no. 2, pp. 148-51, 2006.

[19] S. H. Chang, T. Yasui, S. Taketomi, T. Matsumoto, J. R. KimKaneyama, T. Omiya, Y. Hosaka, H. Inui, Y. Omata, R. Yamagami, D. Mori, F. Yano, U. Chung, S. Tanaka, T. Saito, "Comparison of mouse and human ankles and establishment of mouse ankle osteoarthritis models by surgically-induced instability Osteoarthritis and Cartilage," vol.24, no. 4, April 2016, pp. 688-697.

[20] A. Sutapun and V. Sangveraphunsiri, "A 4-DOF upper limb exoskeleton for stroke rehabilitation: kinematics mechanics and control," International Journal of Mechanical Engineering and Robotics Research, vol. 4, no. 3, pp. 269-272, July 2015.

[21] J. Yoon, J. Ryu, "A novel reconfigurable ankle/foot rehabilitation robot," International Conference on Robotics and Automation IEEE, Barcelona, Spain, pp. 2290-2295, April 2005.

[22] J. Saglia, N. Tsagarakis, J. Dai, and D. Caldwell, "Control strategies for ankle rehabilitation using a high performance ankle exerciser," IEEE International Conference on Robotics and Automation, Alaska, USA, pp. 22212227, May 2010.

[23] J. C. Christensen, J. M. Schuberth, Eric G. Powell Talolisthesis in end stage ankle arthrosis Foot and Ankle Surgery, vol. 22, no. 3, September 2016, pp. 200-204.

[24] H. Y. Hsiao, B. A. Knarr, J. S. Higginson, S. A. Binder-Macleod, "The relative contribution of ankle moment and trailing limb angle to propulsive force during gait Human Movement Science," vol. 39, February 2015, pp. 212-221.

[25] D. Fong, Y. Chan, Y. Hong, P. Yung, K. Fung, and K. Chan, "A three pressure sensor (3PS) system for monitoring ankle supination torque during sport motions," J. Biomech., vol. 41, pp. 2562-2566, 2008.

[26] P. Martin, M. R. Emami, "A neuro-fuzzy approach to real-time trajectory generation for robotic rehabilitation," Robot Autonom Syst, vol. 62, no. 4, PP. 568-78, 2014. 
[27] M. S. Ju, C. C. K. Lin, D. H. Lin, I. S. Hwang, S. M. Chen, "A rehabilitation robot with force-position hybrid fuzzy controller: hybrid fuzzy control of rehabilitation robot," IEEE Trans Neural Syst Rehabil Eng, vol. 13, no. 3, pp. 349-58, 2005.

[28] S. Hussain, S. Q. Xie, P. K. Jamwal, "Control of a robotic orthosis for gait rehabilitation," Robot Autonom Syst; vol. 61, no. 9, pp. 911-19, 2013.

[29] E. Akdogan, M. A. Adli, "The design and control of a therapeutic exercise robot for lower limb rehabilitation: physiotherabot," Mechatronics, vol. 21, no. 3, pp. 509-22, 2011.

[30] J. Espregueira-Mendes, R. B. Pereira, A. Monteiro, Lower Limb Rehabilitation, Springer, 2011, pp. 485-95.

[31] H. Askari, E. Asadi, Z. Saadatnia, A. Khajepour, M. B. Khamesee, J. Zu, "A flexible tube-based triboelectric-electromagnetic sensor for knee rehabilitation assessment," Sensors and Actuators A: Physical, vol. 279, 2018, pp. 694-704,

[32] F. Aggogeri, A. Borboni, N. Pellegrini, "Jerk trajectory planning for assistive and rehabilitative mechatronic devices," International Review of Mechanical Engineering, vol. 10, no. 7, pp. 543-551, 2016.

[33] D. A. Winter, Biomechanics and Motor Control of Human Movement, John Wiley \& Sons, 2009. pp. 45-236.

[34] W. K. Xu, B. Chu, E. Rogers, "Iterative learning control for robotic-assisted upper limb stroke rehabilitation in the presence of muscle fatigue," Control Engineering Practice, vol. 31, October 2014, pp. 63-72.

[35] O. Mendoza-Trejo, C. A. Cruz-Villar, "Modelling and experimental validation of a planar 2-dof cobot as a differentialalgebraic equation system," Applied Mathematical Modelling, volume 40, no. 21-22, November 2016, pp. 9327-9341.

[36] M. Damsgaard, J. Rasmussen, S. T. Christensen, E. Surma, M. de Zee, "Analysis of musculoskeletal systems in the Any Body Modeling System Simulation Modelling Practice and Theory, vol. 14, no. 8, November 2006, pp. 1100-1111

[37] F. Aggogeri, N. Pellegrini, R Adamini, "Functional design in rehabilitation: Modular mechanisms for ankle complex," Applied Bionics and Biomechanics, vol. 2016, 2016, Article ID 9707801

[38] T. Flash, E. Bizzi, "Cortical circuits and modules in movement generation: experiments and theories," Current Opinion in Neurobiology, vol. 41, pp. 174-178, 2016.

[39] H. Choi and J. Ryu, "Development of a tele-rehabilitation system for upper limb using virtual environment and haptics," in Proc. 5 th International Workshop on Human-Friendly Welfare Robotic Systems, KAIST, Daejeon, pp. 68-75, 2004.

[40] M. Zhang, X. Li, "A design of the mechanism for lower limbs passive rehabilitation and kinematics analysis," in Proc. Fifth International Conference On Frontier Of Computer Science And Technology, 2010. pp. 603-7.

[41] P. Culmer, A. Jackson, M. C. Levesley, J. Savage, R. Richardson, J. A. Cozens, et al. "An admittance control scheme for a robotic upper-limb stroke rehabilitation system," in Proc. the IEEE Engineering in Medicine and Biology 27th Conference, 2005, $5081-4$

[42] A. C. Satici, A. Erdogan, and V. Patoglu, "Design of a reconfigurable ankle rehabilitation robot and its use for the estimation of the ankle impedance," in Proc. the IEEE International Conference on Rehabilitation Robotics, (ICORR '09), pp. 257-264, Kyoto, Japan, June 2009.

[43] S. Viñas-Diz, M. Sobrido-Prieto, "Virtual reality for therapeutic purposes in stroke: A systematic review," Neurología, vol. 31, no. 4, May 2016, pp. 255-277.

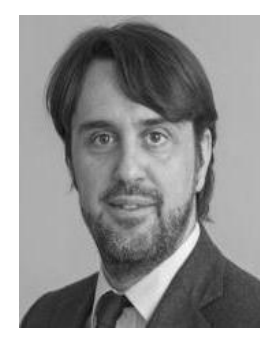

Francesco Aggogeri is an Associate Professor of Applied Mechanics at University of Brescia. His main research interests include Applied Mechanics, Robotics, Mechatronic Devices, Rehabilitation Engineering and Vibration control. He is the author of a number of papers (ISI-Scopus) and peer-review conference proceedings papers. Francesco Aggogeri is responsible of national and international research projects. He is the unit coordinator of PROGRAMS (H2020 project) and he has served as responsible of IntegMicro (EU-FP7 project) and Copernico (EU-FP7 project).

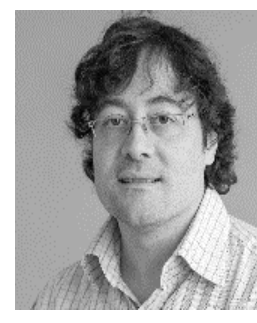

Alberto Borboni was born in Brescia (Italy) in 1973 and received a master degree in Mechanical Engineering in 1997 and PhD in Applied Mechanics in 2012 at the University of Brescia. He is assistant professor of Mechanics for Machines and Mechanical Systems at the same university. He is author of more than a hundred of publications and his research interests are on: mechanical engineering in biomedical applications, smart actuators, kinematics and dynamics of high speed machines, mechanical design of industrial and biomedical systems.

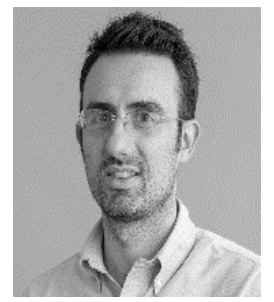

Nicola Pellegrini is an Assistant Professor of Applied Mechanics at University of Brescia. He has been involved in projects related to integrated mechatronic systems, Product development, Motion planning and Soft computing simulation. He is author of a number of papers published in international journals and conferences. He has collaborated to National and European research programs and projects funded by private companies.

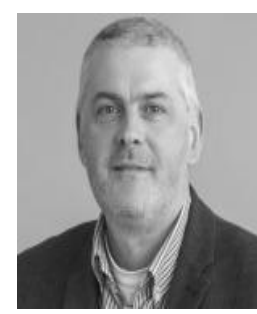

Riccardo Adamini is currently a Full Professor of Applied Mechanics at University of Brescia. $\mathrm{He}$ is author of a number of publications and his research interest includes mechanical engineering, mechanisms and their applications, kinematics and dynamics of industrial systems. 